\title{
Usefulness of thymus and activation-regulated chemokine (TARC) for FPIES diagnosis
}

\author{
Eishi Makita ${ }^{1}$, Daisuke Sugawara ${ }^{1}$, Sae Kuroda $^{2}$, Kae Itabashi $^{1}$, and Ko Ichihashi ${ }^{1}$ \\ ${ }^{1}$ Jichi Ika University Saitama Medical Center \\ ${ }^{2}$ Saitama Red Cross Hospital
}

April 23, 2021

Main Text File

\section{Usefulness of thymus and activation-regulated chemokine (TARC) for FPIES diagnosis Conflicts of interest:}

The authors have no conflicts of interest to declare.

\section{Financial support:}

This study did not avail any funding.

To the Editor:

Food protein-induced enterocolitis syndrome (FPIES), a non-immunoglobulin E-dependent allergic disease, is diagnosed based on clinical symptoms or positive results of an oral food challenge (OFC) test. ${ }^{1,2}$ The lack of disease-specific biomarkers render early diagnosis difficult. Solid food FPIES often develops 5-12 months after birth and may be misdiagnosed as various infectious diseases that are common during that period. Therefore, it is necessary to establish new biomarkers for FPIES diagnosis. In this study, we measured serum thymus and activation-regulated chemokine (TARC) ${ }^{3}$ and general biomarkers in the acute phase of FPIES, infectious gastroenteritis, and sepsis to evaluate the usefulness of each biomarker for FPIES diagnosis. We also examined the relationship between disease severity and serum TARC levels in patients with FPIES.

This case-control study evaluated 20 episodes (9 emergency outpatient visits and 11 positive oral food challenges) of 13 pediatric patients with FPIES ( 8 egg yolk, 1 egg white, 1 whole egg, 1 wheat, 1 rice, and 1 short-neck clam) (FPIES group) and 40 age-matched patients with infectious gastroenteritis or sepsis (control group) between April 2019 and March 2021 (Figure S1, Table S1_Supporting Information). This study was approved by the Ethics Review Committee of the Saitama Medical Center, Jichi Medical University (approval number S19-077). Written consent was obtained from the patient's parents.

Patient background was evaluated. In patients with FPIES, the causative antigen, age of onset, age at event, symptoms, and severity (SuppInfo) of each episode were evaluated.

Serum TARC levels were quantified using a chemiluminescent enzyme immunoassay via a HISCL@ TARC Assay Kit (Sysmex, Hyogo, Japan). For patients who visited the emergency department, serum TARC values were measured at the time of outpatient visit. For OFC cases, serum TARC values were measured before antigen ingestion and at $6 \mathrm{~h}, 24 \mathrm{~h}$, and 1 week after ingestion. Peak serum TARC values were evaluated. Since normal TARC values differ by age, modified TARC values (serum TARC value - normal mean for each age $^{4}$ ) also were evaluated (SuppInfo). Concurrently, complete blood count (CBC) including white blood cell, neutrophil, eosinophil, and platelet, C-reactive protein (CRP), lactate dehydrogenase, venous blood pH, 
$\mathrm{HCO}_{3-}$, and methemoglobin (\%) were measured. OFC was conducted via the open, single-ingestion method (SuppInfo).

The patients with FPIES were five boys and eight girls. The age at onset ranged 0.5-3.0 (median 0.7) years. In the FPIES group, the age for 20 episodes analyzed was $0.5-6.2$ (median 1.1) years. No patient had eczema at the time of the episode. Of these, 7 were severe, 8 were moderate, and 5 was mild in severity (Table 1 ). The control group had 20 patients with infectious gastroenteritis and 20 patients with sepsis aged 0.1-6.0 (median 1.5) years. There were no cases of eczema (Table S1_SuppInfo).

Table 2 shows the blood test results of the FPIES and control groups. The serum TARC $(\mathrm{pg} / \mathrm{mL})$ and modified TARC $(\mathrm{pg} / \mathrm{mL})$ levels were significantly higher in the FPIES group than in the control group $(p$ $<0.001$; Figure 1A). The eosinophil count was also significantly higher in the FPIES group. There were no significant differences regarding other parameters between the groups.

The results of the receiver operating characteristic analysis for each parameter for FPIES diagnosis are shown in Figure 1B and Table S3_SuppInfo. The area under the curve (AUC) of the serum TARC was 0.946 (95\% confidence interval [CI], 0.871-1.000), with a 95\% sensitivity, 95\% specificity, and a cutoff value of 1062 $\mathrm{pg} / \mathrm{mL}$ (Youden Index). The AUC of serum TARC and modified TARC values were higher than those of other parameters.

In univariate analysis, serum TARC levels were a significant factor for FPIES diagnosis, with an odds ratio (OR) of 29.5 (95\% CI 4.3-200.0, $p<0.001)$ per $1000 \mathrm{pg} / \mathrm{mL}$ increase. The age-adjusted TARC OR per 1000 $\mathrm{pg} / \mathrm{mL}$ increase was 23.4 (95\% CI 3.3-162.0, $p=0.001$ ).

The median (range) serum TARC levels for patients with severe $(\mathrm{n}=7)$, moderate $(\mathrm{n}=8)$, and mild $(\mathrm{n}=5)$ FPIES were 6320 (2911-8147), 2020 (1084-4097), and 1087 (410-1678), respectively. Significant differences were found between the severe, moderate, and mild groups ( $p=0.007, p=0.037$ ) (Figure 3C). The correlation coefficients between the serum TARC value and number of vomiting episodes and vomiting time were $R=0.570(p=0.008)$ and $R=0.699(p<0.001)$, respectively, showing a moderate correlation (Figure S2).

Figure 1D shows the transition of the 11 OFC-positive TARC values. The median (range) serum TARC $(\mathrm{pg} / \mathrm{mL})$ was 555 (227-1702) before ingestion, 1283 (410-3821) after $6 \mathrm{~h}$, and 3748 (886-7816) after $24 \mathrm{~h}$, increasing significantly over time. After one week, the median (range) TARC decreased to 961 (468-3359).

This study revealed that serum TARC levels are more useful than other biomarkers, such as CBC, CRP, and methemoglobin levels for FPIES diagnosis. We also found a correlation between the severity of FPIES and serum TARC.

In a previous study comparing acute phase biomarkers in patients with FPIES, infectious gastroenteritis, and sepsis, patients with FPIES had higher white blood cell, eosinophil, platelet counts, and lower CRP levels. However, the usefulness of these biomarkers for FPIES diagnosis is unclear. ${ }^{5}$ We reported that neonates with FPIES had significantly higher methemoglobin levels than those with other diseases with vomiting. Those results were limited to the neonatal period with less overlap of methemoglobin levels in both groups. ${ }^{6}$ There are reports measuring various cytokines, although specific recommendations have not been determined. ${ }^{1}$

TARC is a Th2 type chemokine produced by dendritic cells, endothelial cells, keratinocytes, and fibroblasts. ${ }^{3}$ Although TARC is used in diagnosing and assessing atopic dermatitis, some reports associate it with food allergies. ${ }^{4,7,8}$ Recently, we have evaluated serum TARC levels of the acute phase in patients with FPIES and infectious gastroenteritis and reported that FPIES exhibits high TARC levels. ${ }^{9}$ However, because the control group was small and included patients with infectious gastroenteritis alone, the predictive accuracy could not be clarified. Here, we clarified that serum TARC value is useful for FPIES diagnosis.

A longitudinal evaluation of TARC levels showed an increase at $6 \mathrm{~h}$ post-ingestion, with a further increase $24 \mathrm{~h}$ later. After one week, serum TARC levels had decreased, although they had not normalized. Therefore, TARC measurement after $24 \mathrm{~h}$ is recommended for the diagnosis of FPIES. Furthermore, serum TARC levels 
in patients with FPIES were positively correlated with symptom severity, vomiting frequency, and vomiting duration. Since there were no test items that reflect FPIES severity, we expect that the TARC value after vomiting may be used as an objective evaluation item for FPIES severity.

This study had some limitations. First, all participants had acute solid food FPIES. Patients with cow's milk FPIES or chronic FPIES were excluded. Further studies on these are needed. Second, our sample size was small. Nonetheless, there was a significant difference in the TARC levels between patients with FPIES and the controls, indicating that TARC level elevation is specific to FPIES.

In conclusion, post-emetic serum TARC levels are useful for FPIES diagnosis. Early diagnosis may be possible by measuring TARC after vomiting. It may also be useful for objective severity assessment. Further larger-scale research is needed.

Keywords: biomarkers; food allergy; food protein-induced enterocolitis syndrome; infectious gastroenteritis; pediatrics; sepsis

\section{Signature:}

Eishi Makita ${ }^{1 *}, \mathrm{MD}$, Daisuke Sugawara ${ }^{1}, \mathrm{MD}, \mathrm{PhD}$, Sae Kuroda ${ }^{2}, \mathrm{MD}$, Kae Itabashi $^{1}, \mathrm{MD}$, Ko Ichihashi $^{1}$ $\mathrm{MD}, \mathrm{PhD}$

${ }^{1}$ Department of Pediatrics, Saitama Medical Center Jichi Medical University, Saitama, Japan

${ }^{2}$ Department of Pediatrics, Saitama Red Cross Hospital, Saitama, Japan

*Corresponding author:

Dr. Eishi Makita, Department of Pediatrics, Saitama Medical Center Jichi Medical University, 1-847 Amanuma-cho, Omiya-ku, Saitama 330-8503, Japan

\section{Acknowledgments}

We thank all of the pediatricians and nurses at the Saitama Medical Center, Jichi Medical University, Saitama, Japan. We would like to thank Editage (www.editage.jp) for English-language editing.

\section{Key message}

Solid food protein-induced enterocolitis syndrome (FPIES) often develops 5-12 months after birth and may be misdiagnosed as various infectious diseases that are common during that period. So far, there have been no biomarkers for FPIES, although this study shows a clear increase in certain serologic markers, particularly thymus and activation-regulated chemokine (TARC) levels, especially $24 \mathrm{~h}$ post-ingestion of antigen. This indicates that TARC levels may be of use for the diagnosis of FPIES, although further research is still needed.

\section{References}

1. Nowak-Wegrzyn A, Berin MC, Mehr S. Food protein-induced enterocolitis syndrome. J Allergy Clin Immunol Pract 2020;8:24-35.

2. Nowak-Węgrzyn A, Chehade M, Groetch ME, et al. International consensus guidelines for the diagnosis and management of food protein-induced enterocolitis syndrome: Executive summary-Workgroup Report of the Adverse Reactions to Foods Committee, American Academy of Allergy, Asthma \& Immunology. J Allergy Clin Immunol 2017;139:1111-1126.e4.

3. Knipping K, Knippels LM, Dupont C, Garssen J. Serum biomarkers for allergy in children. Pediatr Allergy Immunol 2017;28:114-123.

4. Fujisawa T, Nagao M, Hiraguchi Y, et al. Serum measurement of thymus and activation-regulated chemokine/CCL17 in children with atopic dermatitis: elevated normal levels in infancy and age-specific analysis in atopic dermatitis. Pediatr Allergy Immunol 2009;20:633-641. 
5. Lee E, Barnes EH, Mehr S, Campbell DE. Differentiating acute food protein-induced enterocolitis syndrome from its mimics: a comparison of clinical features and routine laboratory biomarkers. J Allergy Clin Immunol Pract 2019;7:471-478.e3.

6. Makita E, Kuroda S, Sato H, et al. Comparison of methemoglobin levels in food protein-induced enterocolitis syndrome and other gastrointestinal diseases in neonates. Allergol Immunopathol 2020;48:490-495.

7. Furue M, Matsumoto T, Yamamoto T, et al. Correlation between serum thymus and activation-regulated chemokine levels and stratum corneum barrier function in healthy individuals and patients with mild atopic dermatitis. J Dermatol Sci 2012;66:60-63.

8. Hijnen D, De Bruin-Weller M, Oosting B, et al. Serum thymus and activation-regulated chemokine (TARC) and cutaneous T cell- attracting chemokine (CTACK) levels in allergic diseases: TARC and CTACK are disease-specific markers for atopic dermatitis. J Allergy Clin Immunol 2004;113:334-340

9. Makita E, Kuroda S, Itabashi K, Sugawara D, Ichihashi K. Evaluation of the diagnostic accuracy of thymus and activation-regulated chemokine to discriminate food protein-induced enterocolitis syndrome from infectious gastroenteritis. Int Arch Allergy Immunol 2020;182:229-233.

Table 1. Details of FPIES episodes

\begin{tabular}{ll}
\hline & All episodes $(\mathrm{n}=20)$ \\
\hline $\begin{array}{l}\text { Median age, years (range) } \\
\text { Presentation (n, \%) }\end{array}$ & $1.1(0.5-6.2)$ \\
Emergency department visit & $9(45)$ \\
Oral food challenge test & $11(55)$ \\
Causative food (n, \%) & \\
Egg yolk & $13(65)$ \\
Egg White & $1(5)$ \\
Whole egg & $1(5)$ \\
Wheat & $1(5)$ \\
Rice & $3(15)$ \\
Short-neck clam & $1(5)$ \\
Median time to onset of symptoms, hours (range) & $2.5(1.5-4)$ \\
Symptom & \\
Vomiting, n (\%) & $20(100)$ \\
Median number of vomiting episodes (range) & $5(1-10)$ \\
Median vomiting duration, hours (range) & $3(0.5-10)$ \\
Diarrhea, n (\%) & $5(25)$ \\
Bloody stool, n (\%) & $0(0)$ \\
Lethargy, n (\%) & $15(75)$ \\
Pallor, n (\%) & $8(40)$ \\
Severity, n (\%) & \\
Severe & $7(35)$ \\
Moderate & $8(40)$ \\
Mild & $5(25)$ \\
Treatments, n (\%) & \\
Intravenous fluid & $16(80)$ \\
Intravenous hydrocortisone & $9(45)$ \\
\hline
\end{tabular}

FPIES, food protein-induced enterocolitis syndrome

Table 2 . Comparison of laboratory findings between the FPIES and control groups 


\begin{tabular}{llll}
\hline & FPIES group $(\mathrm{n}=20)$ & Control group $(\mathrm{n}=40)$ & $p$-value \\
\hline TARC $(\mathrm{pg} / \mathrm{mL})$ & $2053(410-8147)$ & $502(104-2034)$ & $<0.001$ \\
Modified TARC $(\mathrm{pg} / \mathrm{mL})$ & $1346(0-7440)$ & $0(0-1327)$ & $<0.001$ \\
WBC $(/ \mu \mathrm{L})$ & $14085(5190-35050)$ & $11205(3360-24060)$ & 0.095 \\
Neutrophils $(/ \mu \mathrm{L})$ & $8578(934-24114)$ & $7046(1584-17684)$ & 0.176 \\
Eosinophils $(/ \mu \mathrm{L})$ & $203(0-832)$ & $0(0-992)$ & 0.006 \\
Platelets $\left(\times 10^{4} / \mathrm{mm}^{3}\right)$ & $36.1(20.7-53.4)$ & $31.7(11.9-58.4)$ & 0.567 \\
$\mathrm{CRP}(\mathrm{mg} / \mathrm{dL})$ & $0.26(0.01-5.92)$ & $0.87(0.01-11.08)$ & 0.083 \\
$\mathrm{LDH}(\mathrm{U} / \mathrm{L})$ & $308(232-383)$ & $298(193-543)$ & 0.270 \\
$\mathrm{pH}$ & $7.380(7.281-7.415)$ & $7.384(7.262-7.510)$ & 0.426 \\
$\mathrm{HCO} 3^{-}(\mathrm{mEq} / \mathrm{L})$ & $19.7(15.4-23.5)$ & $20.9(12.8-26.4)$ & 0.225 \\
Methemoglobin $(\%)$ & $0.4(0.1-0.6)$ & $0.3(0-1.1)$ & 0.075 \\
\hline
\end{tabular}

Data are presented as medians (ranges). Differences were evaluated using the Mann-Whitney $U$ test.

FPIES, food protein-induced enterocolitis syndrome; TARC, thymus and activation-regulated chemokine; WBC, white blood cell count; CRP, C-reactive protein; LDH, lactate dehydrogenase.

Figure legends

\section{Figure 1.}

(A) Comparison of the serum and modified TARC levels between the FPIES and control groups.

The medians and interquartile ranges are indicated by horizontal lines.

(B) Receiver operating characteristic curve for the diagnosis of FPIES.

(C) Comparison of serum TARC and modified TARC between mild, moderate, and severe episodes.

(D) The temporal changes in serum TARC values.

CRP, C-reactive protein; FPIES, food protein-induced enterocolitis syndrome; TARC, thymus and activationregulated chemokine; WBC, white blood cell count

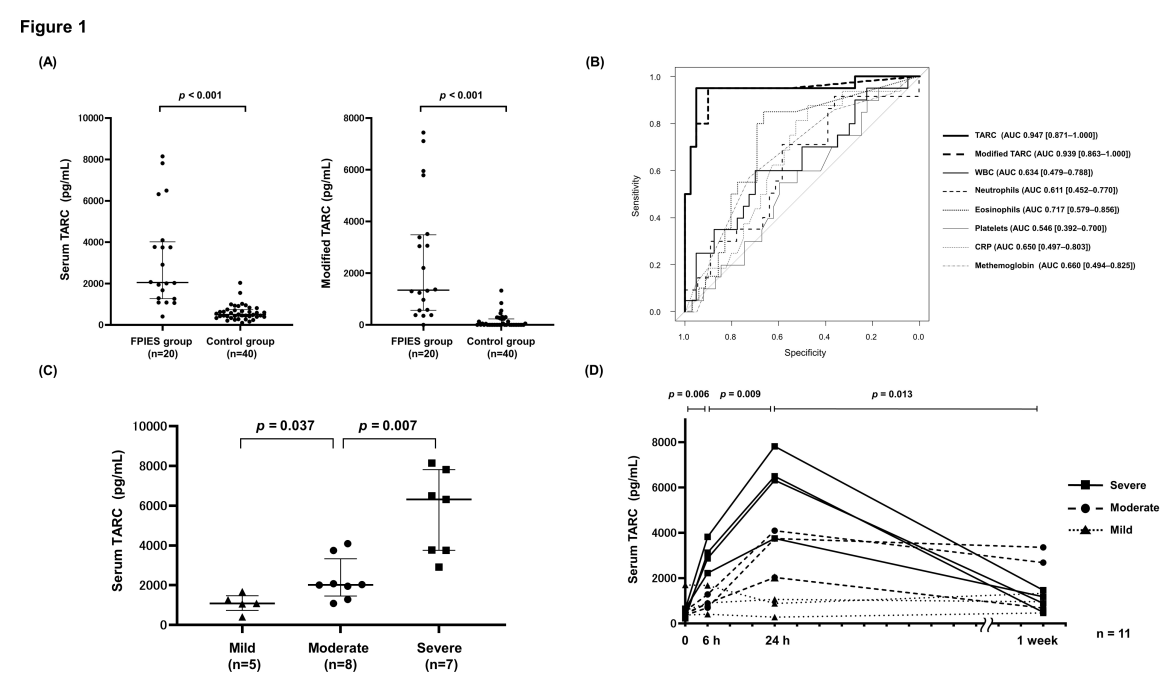

